\title{
A Comparative Study between Analgesic Efficacy of Intrathecal Buprenorphine and USG- Guided Transversus Abdominis Plane Block in Patients Undergoing Cesarean Section under Spinal Anesthesia
}

\author{
Rajashree Deelip Godbole ${ }^{1} \quad$ Abhilash Bandari $^{1} \quad$ Jasmeet Gill $^{1} \quad$ Shruti Tolambia ${ }^{1} \quad$ Yuvraj Hake \\ Pratima Nagare ${ }^{1}$ \\ ${ }^{1}$ Department of Anaesthesiology, K E M Hospital, Rasta Peth, Pune, \\ Address for correspondence Rajashree Deelip Godbole, MD, \\ Maharashtra, India \\ Department of Anaesthesiology, K E M Hospital, Rasta Peth, Pune \\ Int J Recent Surg Med Sci 2022;8:70-77. \\ 411011, Maharashtra, India (e-mail: rajashree.godbole@gmail.com).
}

\begin{abstract}
Objective The aim of this study was to evaluate and compare the postoperative analgesic efficacy of intrathecal buprenorphine with ultrasonography (USG)-guided transversus abdominis plane (TAP) block in patients of cesarean section.

Materials and Methods: Sixty American Society of Anesthesiologists grade I and II pregnant women aged between 20 and 40 years requiring lower segment cesarean section were included in this study.

Study Design A prospective randomized comparative study.

Study Groups Group A received intrathecal hyperbaric bupivacaine $0.5 \% 1.8 \mathrm{~mL}$ with $60 \mu \mathrm{g}$ buprenorphine. Group B received intrathecal hyperbaric bupivacaine $0.5 \% 1.8 \mathrm{~mL}$ with $0.2 \mathrm{~mL}$ sterile normal saline and at the end of surgery USG-guided bilateral TAP block was given with $20 \mathrm{~mL} 0.25 \%$ bupivacaine on each side of abdomen.

Results The mean \pm standard deviation of time to first rescue analgesia in Group A and group B was $9.17 \pm 0.64$ hours and $5.59 \pm 0.50$ hours, respectively. Distribution of mean time to first rescue analgesia among the cases studied is significantly higher in

Keywords

- intrathecal buprenorphine

- postcesarean section analgesia

- TAP block group A compared with group B. The distribution of paracetamol and tramadol requirement in first 24 hours among the cases studied was significantly higher in group B compared with group A.

Conclusion The addition of buprenorphine to intrathecal hyperbaric bupivacaine has advantages over USG-guided TAP block for postoperative analgesia after cesarean section-longer duration of postoperative analgesia, lower analgesic requirements over first 24 hours, and cost-effectiveness.
\end{abstract}

DOI https://doi.org/ 10.1055/s-0041-1740055. ISSN 2455-7420.

\footnotetext{
(c) 2022. Medical and Surgical Update Society. All rights reserved. This is an open access article published by Thieme under the terms of the Creative Commons Attribution-NonDerivative-NonCommercial-License, permitting copying and reproduction so long as the original work is given appropriate credit. Contents may not be used for commercial purposes, or adapted, remixed, transformed or built upon. (https://creativecommons.org/ licenses/by-nc-nd/4.0/)

Thieme Medical and Scientific Publishers Pvt. Ltd., A-12, 2nd Floor, Sector 2, Noida-201301 UP, India
} 


\section{Introduction}

All patients undergoing surgical intervention are anxious about the postoperative pain. Patients undergoing cesarean delivery are a special class who should be given good pain relief to enable the mother to take care of her baby. Acute postoperative pain is maximum for first 48 hours and is moderate-to-severe type of pain. Inadequately treated pain can lead to a broad range of consequences like prolonged recovery, increased morbidity-both physical and psychological, prolonged use of opioids and analgesics, delayed mobilization leading to other complications like deep vein thrombosis and embolization, increased medical costs, and development of chronic pain. ${ }^{1}$

It must always be preemptive analgesia or preventive analgesia. ${ }^{2}$ Multimodal analgesia or balanced analgesia is the recommended technique for postcesarean section pain relief. It combines drugs from different classes like nonsteroidal anti-inflammatory drugs, opioids and analgesic techniques like neuraxial block with opioid, epidural analgesia, local infiltration of the wound, and regional blocks. These techniques target different mechanisms of pain leading to their synergistic actions relieving maximum pain in low doses, thereby reducing the risks of adverse drug effects. ${ }^{3}$ The analgesic regimen needs to meet the goals of providing safe, effective analgesia, with minimal side effects for the mother and her child. Achieving good pain relief after cesarean delivery is challenging because of the altered physiology and possibility of transmission of drugs through breast milk. A pain free mother can take care of her newborn and breast-feed effectively. ${ }^{4}$ Pain experienced after abdominal surgery is primarily contributed by the incision made in the abdominal wall. Pain following cesarean delivery has two components-somatic pain arising from abdominal wound has superficial and deep components and visceral from visceral and uterine manipulations. The somatic pain is transmitted within the anterior divisions of the spinal segmental nerves T10-L1. Visceral uterine nociceptive stimuli ascend through inferior hypogastric plexus and enter the spinal cord through T10-L1 spinal nerves. ${ }^{5}$ Transverses abdominis plane (TAP) block anesthetizes somatic supply of the anterior abdominal wall, which arises from the anterior rami of spinal nerves and runs along the plane between internal oblique muscle and transversus abdominis muscle. The lateral abdominal wall consists of three muscle layers, the external oblique, the internal oblique, and the transversus abdominis and their fascial sheaths. The central abdominal wall also includes the rectus abdominis muscles and its fascial sheath. ${ }^{6}$ Regional anesthetic techniques and nerve blocks is an ideal combination for effective pain management in the perioperative period, either sole or in combination with systemic analgesics. Its use has been shown to decrease postoperative complications like stress response, nausea, vomiting, complications associated with the use of opioids and enhance recovery and helps in early mobilization of patients. TAP block is an easy and safe regional analgesic technique. The technique was first introduced by Rafi in 2001 as a traditional blind landmark technique using the lumbar triangle of petit. ${ }^{7}$ It was further developed by McDonnell et al. ${ }^{8}$ This technique involves the instillation of the local anesthetic agent into a fascial plane between the internal oblique muscle and transversus abdominis muscle, either by landmark technique or under ultrasonography (USG) guidance. USG-guided block has been recently described as a technique that promises better localization and drug deposition. TAP block is a regional analgesic technique that blocks T6-L1 nerve branches and has an evolving role in postoperative analgesia for lower abdominal surgeries. Spinal or epidural anesthesia, coadministered with neuraxial opioids, remains a popular and effective technique in providing anesthesia and analgesia for women undergoing cesarean delivery. Regional anesthetic techniques and nerve blocks have been used for effective pain management in the perioperative period. ${ }^{6-8}$ USG guidance offers the advantage of direct visualization of the needle and of the placement of local anesthetic, which might improve safety and efficacy. ${ }^{9}$

Buprenorphine is a centrally acting synthetic analgesic derived from opiate alkaloid thebaine and possesses high-binding affinity for opioid receptors. Buprenorphine shares some general preclinical and clinical attributes of standard opioid agonists such as morphine and fentanyl but differs by having slow receptor dissociation kinetics, a biphasic ("bell" or "inverted U" shaped) dose-response relation, and a ceiling effect on respiratory depression, but not analgesia, in humans. ${ }^{10,11}$ Buprenorphine is a mixed agonist-antagonist opioid with high affinity at both $\mu$ and kappa opiate receptors. It is an effective analgesic. It has got high molecular weight (481), high lipophilicity, and high affinity for opiate receptors that limit cephalad spread and likelihood of delayed respiratory depression. It is $\sim 25$ times more potent than morphine and has a low level of physical dependence. The side effects include nausea, vomiting, pruritus, drowsiness, and respiratory depression. Buprenorphine is a long-acting, promising analgesic by the epidural and intrathecal route. It enhances the sensory blockade of local anesthetic without affecting the sympathetic activity. ${ }^{12,13}$

This study was undertaken to evaluate and compare the postoperative analgesic efficacy of intrathecal buprenorphine with USG-guided TAP block in patients of cesarean section.

\section{Materials and Methods}

\section{Study Population}

Sixty American Society of Anesthesiologists (ASA) grade I and II pregnant women aged between 20 and 40 years requiring lower segment cesarean section were included.

\section{Study Design}

A prospective randomized comparative study.

\section{Study Groups}

Group A received intrathecal hyperbaric bupivacaine $0.5 \%$ $1.8 \mathrm{~mL}$ with $60 \mu \mathrm{g}$ buprenorphine

Group B received intrathecal hyperbaric bupivacaine $0.5 \%$ $1.8 \mathrm{~mL}$ with $0.2 \mathrm{~mL}$ sterile normal saline and at the end of 
surgery USG-guided bilateral TAP block was given with $20 \mathrm{~mL} 0.25 \%$ bupivacaine on each side of abdomen.

\section{Sample Size Calculation}

The sample size was determined by using the effect sizes from the previously published study ${ }^{18}$ and with the help of following formula:

$$
n \text { (Per Group })=2\left[\frac{\left(Z_{\frac{a}{2}}+Z_{\beta}\right) \sigma}{\Delta}\right]^{2}
$$

Where $n=$ Sample size (per group)

$Z \propto / 2=(1.96)$ for $95 \%$ confidence (i.e., $=0.05) .=1.96$

$Z \beta=$ Cutoff value for power $(1-\beta) .=0.8416$

$\boldsymbol{\Delta}=$ Mean difference to be detected (minimum difference) $=7$ minutes (duration of anesthesia).

$\Delta / \sigma=$ Effect size in SD units $=0.73$

Thus, the sample size according to this formula is $29.46 \approx$ 29 (minimum per group), that is, total 58 (minimum).

Sampling technique: Purposive sampling method.

Study design: Prospective randomized comparative study.

\section{Statistical Data Analysis}

The data on categorical variables will be presented as $n$ (\% of cases) and the values on continuous variables will be presented as mean \pm standard deviation (SD) across two study groups. The significance of the difference of distribution of categorical variables across three study groups will be tested using the chi-squared test. An independent sample $t$-test will be used to test the statistical comparison of the average of continuous variables across two groups. The underlying assumption of normality will be tested before subjecting the study variables to $t$-test.

p-Values less than 0.05 will be statistically significant. All the hypotheses will be formulated using two tailed alternatives against each null hypothesis (hypothesis of no difference). The entire data will be statistically analyzed using Statistical Package for Social Sciences (SPSS ver 21.0, IBM Corporation) for MS Windows.

\section{Inclusion Criteria}

Patient consent, ASA grade I and II, type of surgery, lower segment cesarian section, abdominal hysterectomy, and age between 20 and 40 years were the inclusion criteria.

\section{Exclusion Criteria}

Body mass index $>35$, history of major systemic disease (ASA grade III or more), history of chronic pain, abuse of drugs or alcohol, and history of allergies to any study medications were the inclusion criteria.

Investigations: Complete hemogram, urine examination, and blood grouping and cross matching, wherever required liver function tests, electrocardiogram (ECG), chest X-ray, and two-dimensional echocardiography were done.

\section{Methodology}

All patients were explained the procedure and informed consent was taken 1 day prior to surgery while doing preanesthetic evaluation and were advised fasting 6 hours for solids and 2 hours for clear liquids. Patients were randomly allocated to one of the two groups by a computergenerated randomization.

On the day of surgery in the operating room, an intravenous (IV) line was secured with the 20-gauge cannula. Patients were preloaded with $15 \mathrm{~mL} / \mathrm{kg}$ of Ringer's lactate solution.

Premedication-Inj. ondansetron $4 \mathrm{mg}$ diluted in normal saline and inj. glycopyrrolate $5 \mu \mathrm{g} / \mathrm{kg}$ were given IV slowly.

All ASA standard monitors were attached that included a pulse oximeter in which ECG, noninvasive blood pressure and oxygen saturation (SpO2) were noted. All patients received spinal anesthesia in the left lateral position under all aseptic precautions at L3/4 interspace in the midline using a 27G Quincke's spinal needle.

Group A received intrathecal hyperbaric bupivacaine $0.5 \%$ $1.8 \mathrm{~mL}$ with $60 \mu \mathrm{g}$ buprenorphine

Group B received intrathecal hyperbaric bupivacaine $0.5 \%$

$1.8 \mathrm{~mL}$ with $0.2 \mathrm{~mL}$ sterile normal saline and at the end of surgery USG-guided bilateral TAP block was given with $20 \mathrm{~mL} 0.25 \%$ bupivacaine on each side of abdomen.

Intraoperative hypotension, that is, systolic blood pressure, decreased to $<80 \mathrm{~mm}$ of $\mathrm{Hg}$ or $<20 \%$ of the baseline whichever appeared first and was treated with ephedrine ( $3 \mathrm{mg}$ aliquots) and bradycardia was treated with atropine IV $0.02 \mathrm{mg} / \mathrm{kg}$ if heart rate was less than $60 / \mathrm{min}$. Any adverse events like nausea, vomiting, and pruritis were recorded and treated accordingly.

Postoperatively, the patients were evaluated for hemodynamic parameters, pain, sedation, requirement of other analgesic drugs, complications like nausea, vomiting, and pruritus. Arrival at the postanesthesia care unit was taken as time 0 ; the patients were subsequently assessed at $2,4,6,12$, and 24 hours. The pain was quantified on a $10 \mathrm{~cm}$ visual analog scale (VAS). At the onset of pain, (VAS >3.4) rescue analgesia was given. The VAS is a unidimensional measure of pain intensity that has been widely used in diverse adult populations. On the first request for analgesia, IV paracetamol $1 \mathrm{~g}$ stat was given and repeated every 6 hourly thereafter. Tramadol $50 \mathrm{mg}$ IV was given for breakthrough pain. The time for the first analgesic request and supplemental analgesic requirements over 24 hours were recorded. The sedation level was evaluated using a modified Wilson sedation scale, 1-oriented, 2-drowsy, 3-arousable, and 4-unarousable for 24 hours after surgery. The patients were asked to rate on a three-point scale about their satisfaction with pain management, 1-highly satisfied, 2-satisfied, 3dissatisfied.

\section{Results}

The demographic parameters like age, body mass index, and duration of surgery were comparable in both the groups. The mean heart rate at 4.5 to 7.0 hours among the cases studied was significantly higher in group B compared with group A ( $p$-value $<0.05$ for all). The mean systolic blood pressure at 
5.0 to 6.0 hours was significantly higher in group B compared with group $A$ ( $p$-value $<0.05$ for all). The mean diastolic blood pressure at 5.0 and 5.5 hours was significantly higher in group B compared with group A ( $p$-value $<0.05$ for all). Distribution of mean SpO2 at baseline and in 24 hours among did not differ significantly between the two study groups ( $p$ value $>0.05$ for all) (-Figs. 1-5).

\section{Discussion}

In the review of literature, we found study by Ravindran et al who conducted a study to compare varying doses of intrathecal buprenorphine for postoperative analgesia after ce-

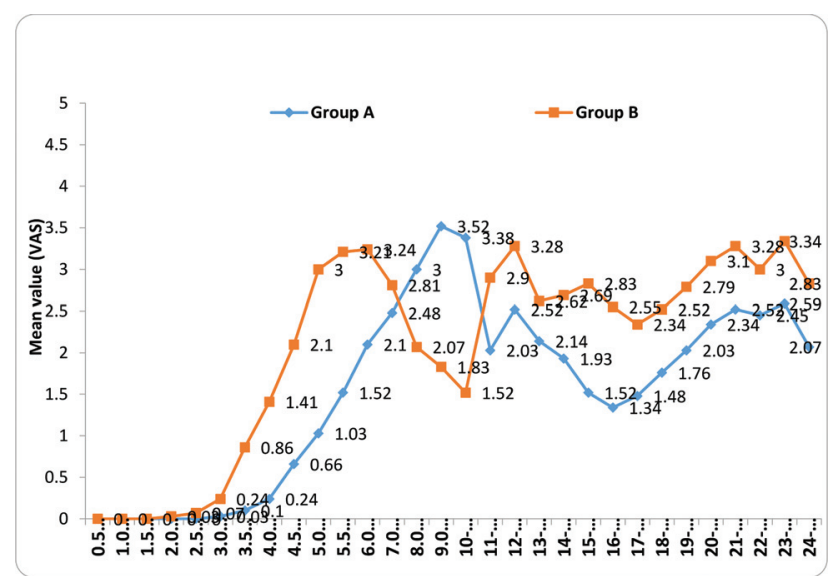

Fig. 1 Intergroup comparison of mean pain score (visual analog scale, VAS). Distribution of mean pain score (VAS) till 2.5 hours among the cases studied did not differ significantly between two study groups ( $p$ value $>0.05$ for all). The mean pain scores (VAS) between 3 hours and 24 hours were significantly higher in group B compared with group $A$ ( $p$-value $<0.05$ for all) except between 8 and 10 hours where the pain scores were high in group A compared with group B.

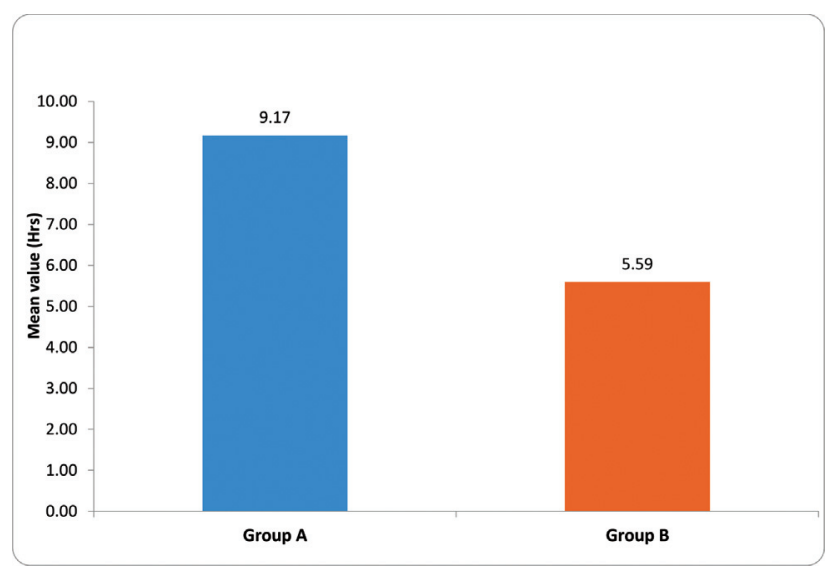

Fig. 2 Intergroup comparison of mean time to first rescue analgesia. The mean \pm standard deviation (SD) of time to first rescue analgesia in group $A$ and group B was $9.17 \pm 0.64$ and $5.59 \pm 0.50$ hours, respectively. The minimum-maximum range of time to first rescue analgesia in group A and group B was 8 to 10 and 5 to 7 hours, respectively. Distribution of mean time to first rescue analgesia among the cases studied is significantly higher in group A compared with group B ( $p$-value $<0.001)$. sarean section. Ninety patients posted for elective section were divided into three groups. Group A received hyperbaric bupivacaine $0.5 \% 1.8 \mathrm{~mL}$ with $45 \mu \mathrm{g}$ buprenorphine and group B received hyperbaric bupivacaine $0.5 \% 1.8 \mathrm{~mL}$ with

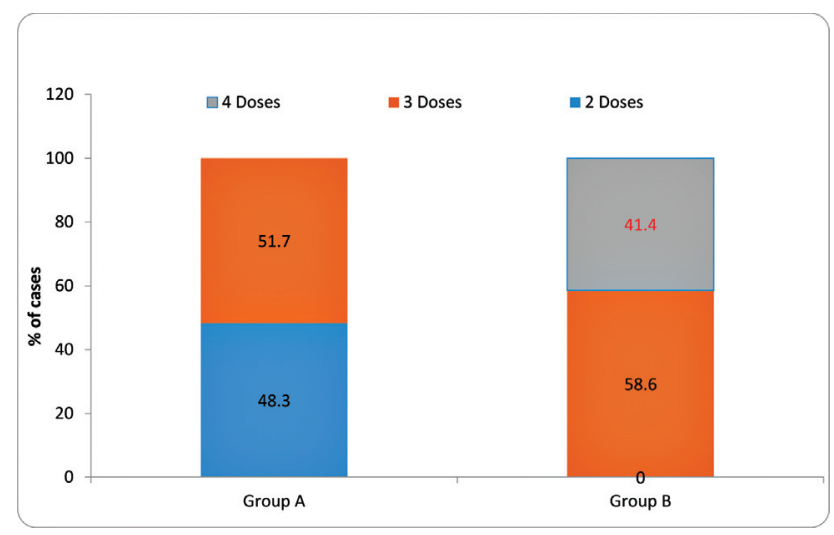

Fig. 3 Intergroup comparison of paracetamol requirement. The distribution of paracetamol requirement first 24 hours among the cases studied was significantly higher in group $B$ compared with group A ( $p$-value $<0.001)$.

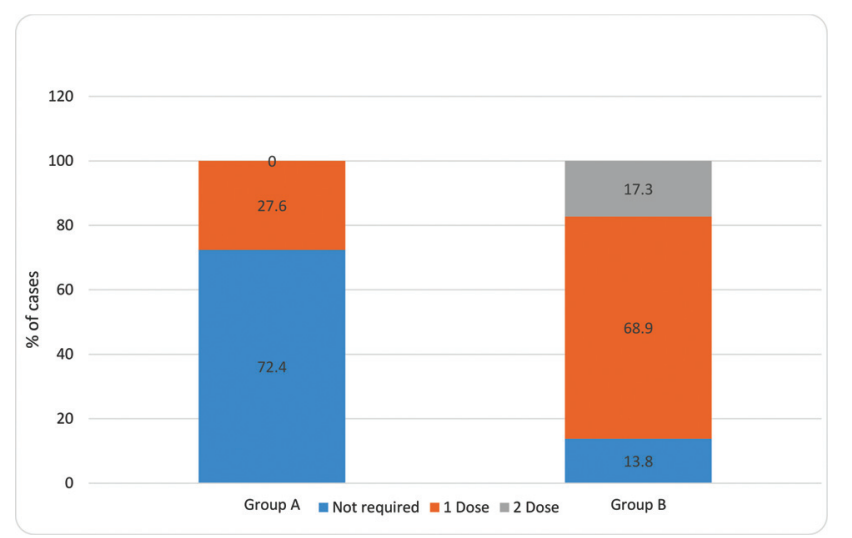

Fig. 4 Intergroup comparison of tramadol requirement in the first 24 hours. The distribution of tramadol requirement in the first 24 hours among the cases studied was significantly higher in group $B$ compared with group A ( $p$-value $<0.001)$.

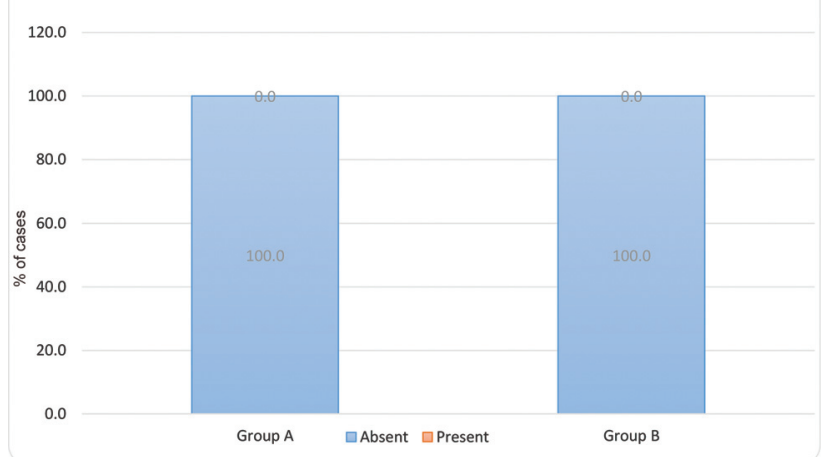

Fig. 5 Intergroup comparison of the incidence of side effects. Of the cases studied in group A and in group B, none had side effects. The distribution of the incidence of side effects among the cases studied did not differ significantly between two study groups ( $p$-value $>0.05$ ). 
$60 \mu \mathrm{g}$ buprenorphine. Group $\mathrm{C}$ received hyperbaric bupivacaine $0.5 \% 1.8 \mathrm{~mL}$ with $0.2 \mathrm{~mL}$ sterile normal saline. Postoperative analgesia was assessed with VAS. Pain at rest and on movement was assessed. Inj. tramadol $1 \mathrm{mg} / \mathrm{kg}$ IV was used as rescue analgesic at VAS score of 4 . The number of doses of rescue analgesia were noted in the first 24 hours.

Duration of analgesia was significantly longer in group A and group B compared with group C. There was significant difference in the postoperative analgesia even in group $A$ (6.1 hours) and group $B$ (12.3 hours) also. Two patients in group A and 5 patients in group B did not require any additional analgesic in the study period. There conclusions were that addition of buprenorphine to hyperbaric bupivacaine prolongs postoperative analgesia without much maternal and neonatal side effects. Increasing the dose of buprenorphine from $45 \mu \mathrm{g}$ to $60 \mu \mathrm{g}$ produced significantly prolonged duration of postoperative analgesia and reduced the requirement of other analgesics without increase in the adverse effects. They concluded that the addition of $60 \mu \mathrm{g}$ buprenorphine to intrathecal hyperbaric bupivacaine is safe, effective, and easy method of postoperative analgesia after cesarean section. ${ }^{14}$ In our study, we have also added $60 \mu \mathrm{g}$ buprenorphine to intrathecal hyperbaric bupivacaine.

Dr Letha J conducted a study to compare intrathecal buprenorphine with bupivacaine and bupivacaine for postcesarean section analgesia. She used $1 \mu \mathrm{g} / \mathrm{kg}$ buprenorphine with $10 \mathrm{mg}$ bupivacaine heavy. The maximum dose of bupivacaine was $60 \mu \mathrm{g}$. Her conclusions are that buprenorphine hastens the onset of analgesia and prolongs the analgesia with mild side effects like nausea, vomiting, and drowsiness. Intrathecal buprenorphine in the dose of $1 \mu \mathrm{g} / \mathrm{kg}$ offers good quality analgesia, is safe, and inexpensive. ${ }^{15}$ The dose of buprenorphine in our study was $60 \mu \mathrm{g}$.

Marappa et al conducted a study to compare the analgesic efficacy of intrathecal buprenorphine (ITB) with USG-guided TAP block for postcesarean delivery analgesia. Sixty patients of ASA physical status I and II posted for elective cesarean section were randomized into two groups of 30 each using sealed envelope method, that is, ITB group and TAP block group. The subarachnoid block was performed with $1.75 \mathrm{~mL}$ $(8.75 \mathrm{mg})$ of $0.5 \%$ of hyperbaric bupivacaine and $75 \mu \mathrm{g}$ $(0.25 \mathrm{~mL})$ of buprenorphine totaling to a volume of $2.0 \mathrm{~mL}$ injected in ITB group and in TAP block group $1.75 \mathrm{~mL}$ $(8.75 \mathrm{mg}$ ) of $0.5 \%$ hyperbaric bupivacaine and $0.25 \mathrm{~mL}$ sterile normal saline totaling to a volume of $2.0 \mathrm{~mL}$ and postoperative USG-guided bilateral TAP block with $20 \mathrm{~mL}$ of $0.25 \%$ bupivacaine on each side without adjuvant. Patients were assessed for the first 24 hours. On the first request for analgesia IV paracetamol $1 \mathrm{~g}$ was given and repeated every 6 hourly thereafter. Tramadol $50 \mathrm{mg}$ IV was given for breakthrough pain. They found that the mean time to first analgesic request was significantly longer in the ITB group (669.17 minutes) compared with the TAP group (389.67 minutes) and was statistically significant. The paracetamol consumption in the first 24 hours was significantly higher in the TAP group as compared with the ITB group, and was statistically significant. Tramadol requirement in the first 24 hours was significantly higher in the TAP group as compared with the ITB group and was statistically significant. The mean VAS scores assessed at 4, 6, 12, and 24 hour were higher in the TAP group and were statistically significant. The incidence of complications like nausea, vomiting, drowsiness, and pruritus was higher in the ITB group compared with the TAP group. The patients in ITB group had much higher satisfaction compared with those in the TAP block group. They concluded from their study that patients receiving ITB for pain management reported a longer duration of analgesia, lower pain scores, and lower analgesic consumption during the first 24 hours. ${ }^{16}$ In our study, the spinal dose of 0.5 bupivacaine heavy was $1.8 \mathrm{~mL}$ with $0.2 \mathrm{~mL}$ buprenorphine or $0.9 \%$ sterile normal saline. Our results are comparable to this study.

Costello et al conducted a study- the TAP block when used as a part of a multimodel regimen inclusive of intrathecal morphine does not improve analgesia after cesarean delivery. Ninety-six patients scheduled for cesarean section under spinal anesthesia were divided into two groups. Forty-nine patients were allocated to the placebo group and 47 patients were allocated to the study group. All patients received standard spinal anesthesia consisting of $12 \mathrm{mg}$ of hyperbaric $0.75 \%$ bupivacaine with $10 \mu \mathrm{g}$ of fentanyl and $100 \mu \mathrm{g}$ of morphine. Bilateral USG-guided TAP block was performed at the end of the surgery with ropivacaine $0.375 \% 20 \mathrm{~mL}$ on each side in the abdomen in the study group and $20 \mathrm{~mL}$ saline on each side in the placebo group. Each patient was assessed at $6,12,24$, and 48 hours postoperatively. Patients received IV $30 \mathrm{mg}$ of ketorolac and $1.3 \mathrm{~g}$ of rectal acetaminophen at the end of the surgical procedure. The standardized postoperative analgesic regimen consisted of $50 \mathrm{mg}$ of oral diclofenac every 8 hourly and $1 \mathrm{~g}$ of oral acetaminophen every 6 hourly for the first 48 hours postoperatively. Morphine was given for breakthrough pain. They found that VAS pain score with movement at 24 hours was $3.2(2.2) \mathrm{cm}$ for the placebo group and $3.4(2.4) \mathrm{cm}$ for the study group. This was not statistically significant. The VAS pain scores at rest were $\sim 50 \%$ of those with movement; there was no difference between the study and the placebo groups. The number of patients requiring additional opioids and the number of opioids consumed did not differ between groups. They concluded from their study that TAP block when used as part of a multimodal regimen inclusive of intrathecal morphine does not improve the quality of postcesarean analgesia. ${ }^{17}$

Kanazi et al conducted a study to compare the analgesic efficacy of subarachnoid morphine (SAM) in comparison with USG-guided TAP block after cesarean delivery. Fiftyseven patients posted for cesarean section were randomized into two groups. Subarachnoid block was performed with $0.75 \%$ hyperbaric bupivacaine in combination with $0.2 \mathrm{mg}$ morphine in SAM group and in TAP group $0.75 \%$ hyperbaric bupivacaine with normal saline $(0.2 \mathrm{~mL})$ and postoperative USG-guided bilateral TAP block was performed using saline in SAM group and bupivacaine $0.375 \%$ with epinephrine 5 $\mu \mathrm{g} / \mathrm{mL}$ in TAP group with $20 \mathrm{~mL}$ on each side. At the end of the surgery, patients in both groups received rectal diclofenac $100 \mathrm{mg}$ and IV paracetamol $1 \mathrm{~g}$. Patients were assessed at 2, 4, $6,12,24,36$, and 48 hours. For the first 24 hours, protocol for 
postoperative analgesia consisted of rectal diclofenac $100 \mathrm{mg}$ every 12 hourly and IV paracetamol $1 \mathrm{~g}$ every 6 hourly. For breakthrough pain, patients were treated with IV tramadol $100 \mathrm{mg}$ every 8 hourly as necessary. For the second 24 hours, rectal diclofenac $100 \mathrm{mg}$ every 12 hourly and two tablets of oral paracetamol $500 \mathrm{mg}$ every 6 hourly and IV tramadol $100 \mathrm{mg}$ every 8 hourly were administered upon patient request for breakthrough pain. They found that time to first analgesic request was longer in group SAM than in group TAP ( 8 hours vs. 4 hours $[p=0.005]$ ). The number of tramadol doses received between 0 and 12 hours was $0(0-1)$ in group SAM versus $0(0-2)$ in group TAP $(p=0.03)$. The number of oral paracetamol doses was not different between the two groups. Postoperative visceral pain scores at rest and on movement during first the 4 hours were lower in group SAM than in group TAP. The incidence of moderate-to-severe nausea was higher in group SAM than in TAP group. More patients developed pruritus requiring treatment in group SAM than in group TAP. They concluded from their study that SAM provided superior analgesia when compared with USGguided TAP block after cesarean delivery. ${ }^{18}$

McMorrow et al conducted a study to compare TAP block with spinal morphine for pain relief after cesarean section. Eighty patients posted for elective cesarean delivery were divided into four groups. All patients received spinal anesthesia with $0.5 \%$ hyperbaric bupivacaine 11 to $12.5 \mathrm{mg}$ with fentanyl $10 \mu \mathrm{g}$ and in addition received spinal morphine or spinal saline. All patients were given bilateral TAP block with $0.375 \%$ bupivacaine $2 \mathrm{mg} / \mathrm{kg}$ or equivalent volume of $0.9 \%$ sterile normal saline. All subjects received rectal paracetamol $1 \mathrm{~g}$ and diclofenac $100 \mathrm{mg}$ immediately after the operation. Each patient received bilateral TAP blocks in the operating theater immediately after the completion of surgery. All patients were prescribed a standard postoperative analgesic regime of regular oral paracetamol $1 \mathrm{~g} 6$ hourly, rectal diclofenac $100 \mathrm{mg} 18$ hourly, and morphine via patient-controlled analgesia. They concluded that pain on movement and early morphine consumption was lowest in groups receiving spinal morphine and was not improved by the TAP block. They concluded from their study that spinal morphine and not TAP block improved analgesia after cesarean section. The addition of TAP block to spinal morphine did not further improve analgesia. ${ }^{19}$

Mankikar et al conducted a study on USG-guided TAP block for postoperative analgesia in patients undergoing cesarean section. Sixty ASA I and II patients posted for elective and emergency cesarean section were divided into two groups. All patients received spinal anesthesia with $0.5 \%$ hyperbaric bupivacaine $10 \mathrm{mg}$. Patients were divided into two groups: group S-USG-guided TAP block with 0.5\% ropivacaine $(n=30) 15 \mathrm{~mL}$ on either side and group $\mathrm{C}-$ USG-guided TAP block with $0.9 \%$ normal saline $(n=30)$ $15 \mathrm{~mL}$ on either side. All patients received injection paracetamol $1 \mathrm{~g}$ IV at the end of the surgery. Tramadol $2 \mathrm{mg} / \mathrm{kg}$ was administered as a rescue analgesic IV when VAS was more than four. Time to first analgesic administration (tramadol) was prolonged significantly in group S (mean: 9.53 hours) as compared with group C. In patients receiving TAP block with
$0.5 \%$ ropivacaine (group $\mathrm{S}$ ), the requirement for analgesics was significantly reduced as compared with those who received the placebo block (group $\mathrm{C}$ ). The mean tramadol requirement in group $S$ was $140 \mathrm{mg}$ and in group $\mathrm{C}$ it was $246.66 \mathrm{mg}$, which was statistically significant. VAS was reduced after TAP block with $0.5 \%$ ropivacaine for the first 8 to 10 hours postoperatively as compared with patients receiving a placebo block. They concluded that USG-guided bilateral TAP block with $0.5 \%$ ropivacaine reduces the postoperative opioid analgesic consumption. ${ }^{20}$

John et al conducted a study to compare the analgesic efficacy of TAP block after elective cesarean delivery-bupivacaine with fentanyl versus bupivacaine alone. Sixty patients posted for elective cesarean section were randomized into two groups of 30 each. All patients received a subarachnoid block with $2 \mathrm{~mL}$ of $0.5 \%$ bupivacaine (heavy). Patients were randomly allocated to undergo TAP block (group A) with either $38 \mathrm{~mL}$ of $0.25 \%$ bupivacaine $+50 \mu \mathrm{g}$ of fentanyl or TAP block (group B) with 38 of $0.25 \%$ bupivacaine $+2 \mathrm{~mL}$ of normal saline at the end of surgical procedure. Total volume was divided into two equal doses $(20 \mathrm{~mL}$ each) to be administered on either side. Diclofenac $75 \mathrm{mg}$ intramuscular was given as rescue analgesia. Groups A and B had similar pain scores at $0,1,2,3,4,5,6,8,12$, and 24 hours at rest and on movement. In both groups, the total analgesic requirement did not show any statistical significance. They concluded that the TAP block as a part of a multimodal analgesic regimen has a role in providing superior analgesia in the postoperative period. However, addition of fentanyl to local anesthetic bupivacaine was found to have no added advantage when the quality and duration of analgesia were compared. $^{21}$

Akkaya et al conducted a study on 42 patients of ASA grade I to II posted for cesarean section who were divided into two groups. All patients were given spinal anesthesia with inj. bupivacaine. After the surgery, USG-guided TAP block was given to all patients. Levobupivacaine $0.25 \% 30 \mathrm{~mL}$ with $2 \mathrm{~mL} 0.9 \% \mathrm{NaCl}$ was given bilaterally in one group and levobupivacaine $0.25 \% 30 \mathrm{~mL}$ with $8 \mathrm{mg}$ dexamethasone was given bilaterally in the second group. The time for first rescue analgesia and total consumption of other analgesics were noted. The time for first rescue analgesia was $6.1 \pm 4.8$ hours in plain levobupivacaine group and $13 \pm 7.8$ hours in dexamethasone group. The total consumption of tramadol was significantly low in dexamethasone group. They concluded that addition of dexamethasone in TAP block prolongs the duration of postoperative analgesia after cesarean section and it reduces the consumption of other analgesics in the postoperative period. ${ }^{22}$

Parameswari and Udayakumar conducted a study in 70 ASA I and II patients posted for elective cesarean section. The patients were divided into two groups of 35 each. All patients were given spinal anesthesia for cesarean section. At the end of surgery, all patients received USG-guided TAP block bilaterally. The study group received inj. bupivacaine $0.25 \%$ $20 \mathrm{~mL}$ with $0.5 \mu \mathrm{g} / \mathrm{kg}$ dexmedetomidine and the control group received inj. bupivacaine $0.25 \% 20 \mathrm{~mL}$. The average time at which rescue analgesia was required was 14.25 hours 
in study group and 7.73 hours in control group that was statistically significant. They concluded that addition of dexmedetomidine to bupivacaine in TAP block prolongs the time for first dose of rescue analgesia and reduces the consumption of opioids in the postoperative the first 24 hours. $^{23}$

Mishriky et al did a systematic review and meta-analysis of randomized controlled trials comparing efficacy of TAP block in patients undergoing elective cesarean delivery under spinal anesthesia and reported postoperative consumption of morphine and pain scores. Their conclusions are as follows: TAP block is an effective modality of pain relief in patients undergoing cesarean delivery under spinal anesthesia without morphine. It reduces pain scores, postoperative consumption of morphine, and opiate-related side effects. In patients receiving intrathecal morphine, the analgesia was better but with opioid-related side effects. TAP block just reduces pain scores on movement in the early postoperative period in these patients. ${ }^{24}$

Abdallah et al have done a systematic review and metaanalysis of randomized controlled trials comparing TAP block with placebo trials in patients undergoing elective cesarean delivery under spinal anesthesia. They concluded that TAP block is an effective analgesic for post cesarean delivery under spinal anesthesia when spinal morphine is not used. It reduces the postoperative consumption of morphine in the first 24 hours. When intrathecal morphine is used, TAP block is of no benefit. ${ }^{25}$

\section{Conclusion}

We recommend the addition of buprenorphine to intrathecal hyperbaric bupivacaine compared with USG-guided TAP block for postoperative analgesia after cesarean section for following advantages: longer duration of postoperative analgesia, lower analgesic requirements over first 24 hours, lower pain scores postoperatively, improvement in the quality of pain relief, lower incidence of side effects as compared with other additives, and cost effective. No additional costly equipment like USG machine is required that may not be available at all centers. Addition of dexamethasone in TAP block can prolong the duration of analgesia of TAP block.

\section{Conflict of Interest}

None declared.

\section{References}

1 Gan TJ. Poorly controlled postoperative pain: prevalence, consequences, and prevention. J Pain Res 2017;10:2287-2298

2 Dahl JB, Kehlet H. Preventive analgesia. Curr Opin Anaesthesiol 2011;24(03):331-338

3 Polomano RC, Fillman M, Giordano NA, Vallerand AH, Nicely KL, Jungquist CR. Multimodal analgesia for acute postoperative and trauma-related pain. Am J Nurs 2017;117(3, Suppl 1):S12-S26

4 Ismail S. What is new in postoperative analgesia after caesarean sections? (Editorial). Anaesth Pain Intensive Care 2012;16(02): $123-126$
5 McDonnell NJ, Keating ML, Muchatuta NA, Pavy TJG, Paech MJ. Analgesia after caesarean delivery. Anaesth Intensive Care 2009; 37(04):539-551

6 Miller RD, Cohen NH, Eriksson LI, Fleisher LA, Wiener-Kronish JP, Young WL. Miller's Anesthesia. 8th ed.Philadelphia, PA: Elsevier Saunders; 2015:19103-2899

7 Rafi AN. Abdominal field block: a new approach via the lumbar triangle. Anaesthesia 2001;56(10):1024-1026

8 McDonnell JG, Curley G, Carney J, et al. The analgesic efficacy of transversus abdominis plane block after cesarean delivery: a randomized controlled trial. Anesth Analg 2008;106(01): 186-191

9 Hebbard P, Fujiwara Y, Shibata Y, Royse C. Ultrasound-guided transversus abdominis plane (TAP) block. Anaesth Intensive Care 2007;35(04):616-617

10 Ding Z, Raffa RB. Identification of an additional supraspinal component to the analgesic mechanism of action of buprenorphine. Br J Pharmacol 2009;157(05):831-843

11 Dahan A, Yassen A, Romberg R, et al. Buprenorphine induces ceiling in respiratory depression but not in analgesia. $\mathrm{Br} \mathrm{J}$ Anaesth 2006;96(05):627-632

12 Pharmacology and Physiology in Anaesthesia Practice. 3rd edition. Philadelphia, Pennsylvania: Lippincott-Raven Publishers; 1999:105-106

13 Dixit S. Post operative analgesia after caeasarean section: an experience with intrathecal buprenorphine. Indian J Anaesth 2007;51(06):515-518

14 Ravindran R, Sajid B, Ramadas KT, Susheela I. Intrathecal hyperbaric bupivacaine with varying doses of buprenorphine for postoperative analgesia after cesarean section: a comparative study. Anesth Essays Res 2017;11(04):952-957

15 Dr Letha J. Comparison of intrathecal bupivacaine with or without buprenorphine for postoperative pain relief in caesarean section. JM SC R. 2018;06(08):54-59

16 Marappa P, Chikkapillappa MA, Chennappa NM, Pujari VS. A comparative study of analgesic efficacy of intrathecal buprenorphine with ultrasound-guided transversus abdominis plane block for postcesarean delivery analgesia. Anesth Essays Res 2017;11 (02):376-379

17 Costello JF, Moore AR, Wieczorek PM, Macarthur AJ, Balki M, Carvalho JCA. The transversus abdominis plane block, when used as part of a multimodal regimen inclusive of intrathecal morphine, does not improve analgesia after cesarean delivery. Reg Anesth Pain Med 2009;34(06):586-589

18 Kanazi GE, Aouad MT, Abdallah FW, et al. The analgesic efficacy of subarachnoid morphine in comparison with ultrasound-guided transversus abdominis plane block after cesarean delivery: a randomized controlled trial. Anesth Analg 2010;111(02): 475-481

19 McMorrow RCN, Ni Mhuircheartaigh RJ, Ahmed KA, et al. Comparison of transversus abdominis plane block vs spinal morphine for pain relief after Caesarean section. Br J Anaesth 2011;106(05): 706-712

20 Mankikar MG, Sardesai SP, Ghodki PS. Ultrasound-guided transversus abdominis plane block for post-operative analgesia in patients undergoing caesarean section. Indian J Anaesth 2016; 60(04):253-257

21 John R, Ranjan RV, Ramachandran TR, George SK. Analgesic efficacy of transverse abdominal plane block after elective cesarean delivery - bupivacaine with fentanyl versus bupivacaine alone: a randomized, double-blind controlled clinical trial. Anesth Essays Res 2017;11(01):181-184

22 Akkaya A, Yildiz I, Tekelioglu UY, et al. Dexamethasone added to levobupivacaine in ultrasound-guided transversus abdominis plain block increased the duration of postoperative analgesia after caesarean section: a randomized, double blind, controlled trial. Eur Rev Med Pharmacol Sci 2014;18(05):717-722 
23 Ramya Parameswari A, Udayakumar P. Comparison of efficacy of bupivacaine with dexmedetomidine versus bupivacaine alone for transversus abdominis plane block for post-operative analgesia in patients undergoing elective caesarean section. J Obstet Gynaecol India 2018;68(02):98-103

24 Mishriky BM, George RB, Habib AS. Transversus abdominis plane block for analgesia after cesarean delivery: a systematic review and meta-analysis. Can J Anaesth 2012;59(08): 766-778

25 Abdallah FW, Halpern SH, Margarido CB. Transversus abdominis plane block for postoperative analgesia after Caesarean delivery performed under spinal anaesthesia? A systematic review and meta-analysis. $\mathrm{Br} \mathrm{J}$ Anaesth 2012;109(05): 679-687 